\title{
On the topology and area of higher-dimensional black holes
}

\author{
Mingliang Cai and Gregory J Galloway \\ Department of Mathematics, University of Miami, Coral Gables, FL 33124, USA
}

Received 6 March 2001

Published 28 June 2001

Online at stacks.iop.org/CQG/18/2707

\begin{abstract}
Over the past decade there has been an increasing interest in the study of black holes, and related objects, in higher (and lower) dimensions, motivated to a large extent by developments in string theory. The aim of the present paper is to obtain higher-dimensional analogues of some well known results for black holes in $3+1$ dimensions. More precisely, we obtain extensions to higher dimensions of Hawking's black hole topology theorem for asymptotically flat $(\Lambda=0)$ black hole spacetimes, and Gibbons' and Woolgar's genus-dependent, lower entropy bound for topological black holes in asymptotically locally antide Sitter $(\Lambda<0)$ spacetimes. In higher dimensions the genus is replaced by the so-called $\sigma$-constant, or Yamabe invariant, which is a fundamental topological invariant of smooth compact manifolds.
\end{abstract}

PACS numbers: 0470,1125

\section{Introduction}

Among the most fundamental macroscopic features of a black hole are its topology and area. The first general theorem on the topology of black holes was due to Hawking [19] in the early 1970 s who proved that the boundary surface of a black hole (obtained by intersecting the event horizon with a suitable spacelike hypersurface) in a $(3+1)$-dimensional asymptotically flat stationary black hole spacetime obeying the dominant energy condition is spherical. Much later, during the mid 1990s, it was realized that results on topological censorship could be used to improve various aspects of Hawking's black hole topology theorem (cf [12] and references cited therein). At the same time, during the 1990s, it was recognized that under appropriate circumstances, one could have as black hole boundaries surfaces of higher genus. As is now well known, such examples can occur in $(3+1)$-dimensional black hole spacetimes which are asymptotically locally anti-de Sitter, and which, in particular, have non-trivial topology at infinity (see, e.g., [5], and references cited therein). Such examples, of course, do not violate Hawking's theorem; because of the presence of a negative cosmological constant, the dominant energy condition is not satisfied. These examples, however, do satisfy the mild 
energy conditions of topological censorship, and, in fact, as shown in [12], are consistent with topological censorship. In the general setting of asymptotically locally anti-de Sitter spacetimes, topological censorship implies that the genus of the black hole boundary (or, if there is more than one black hole, the sum of the genera of the black hole boundaries) is bounded above by the genus of the surface at infinity; in particular, if the latter is spherical, so is the black hole boundary. Hence, in $3+1$ dimensions, the topology of black holes is controlled by the topology at infinity.

Although Hawking's theorem does not hold in the asymptotically locally anti-de Sitter setting, his basic argument still applies and leads to an interesting conclusion, as pointed out by Gibbons [16] in the time-symmetric case. In this case, under circumstances closely related to those considered by Hawking, and assuming non-negative local energy density $T_{00} \geqslant 0$, Gibbons obtains the following lower bound for the area of a black hole boundary $\Sigma$ :

$$
\operatorname{Area}(\Sigma) \geqslant \frac{4 \pi(g-1)}{|\Lambda|}
$$

where $g$ is the genus of $\Sigma$, and $\Lambda<0$ is the cosmological constant. Hence, in this situation the black hole entropy has a lower bound depending on a global topological invariant. Shortly thereafter, Woolgar [35] obtained independently a similar inequality in the general (non-timesymmetric) case.

During the past decade there has been a significant increase in interest in black holes in higher (and lower) dimensions, due largely to the recognition of their relevance to string theory (see, for example, the review articles of Horowitz [23] and Peet [29]). There is now an extensive literature of solutions in string theory with horizons, which represent black holes, and related objects, such as black strings and black $p$-branes, in various dimensions. The physical properties of these solutions have been widely studied. Interest in black holes in various dimensions has intensified in recent years, due, for example, to the role they have played in the conjectured correspondence between string theory (or supergravity) on asymptotically locally anti-de Sitter backgrounds and the large- $N$ limit of certain conformal field theories defined on the boundary-at-infinity of these backgrounds, cf [1,27,34].

The aim of the present paper is to establish some general properties of black holes which hold in arbitrary dimension. To be more specific, our goal is to obtain extensions of Hawking's black hole topology theorem (relevant when $\Lambda \geqslant 0$ ) and Gibbon's entropy bound (relevant when $\Lambda<0$ ) to higher dimensions. The precise setting for these results is presented in the next section. We restrict attention primarily to the time-symmetric case. Some comments regarding the general case are given in section 4.

In section 2 we obtain restrictions on the topology of a black hole boundary surface $\Sigma^{n-1}$ sitting in a spacelike hypersurface $V^{n}$ in a spacetime $M^{n+1}, n \geqslant 3$. We show under suitable circumstances (in particular, under appropriate energy conditions) that $\Sigma^{n-1}$ must admit a metric of positive scalar curvature (which need not be the induced metric). In the $3+1(\operatorname{dim} \Sigma=2)$ case, this implies, by Gauss-Bonnet that $\Sigma$ is a 2 -sphere, and we recover Hawking's theorem. The point, however, is that there are many known topological obstructions to metrics of positive scalar curvature in higher dimensions, as well, as we recall in section 2 . In particular, in the $4+1(\operatorname{dim} \Sigma=3)$ case, the topology of $\Sigma$ is restricted to the 3 -sphere (modulo the Poincaré conjecture) perhaps with identifications, $S^{2} \times S^{1}$, and finite connected sums of such manifolds.

The proofs of the results in section 2 are based on a variation of Hawking's original argument. However, his argument does not directly extend to higher dimensions, since, in higher dimensions, the Gauss-Bonnet theorem cannot be used, as it was by Hawking, to control the total scalar curvature of $\Sigma$. Instead, we combine Hawking's argument together 
with arguments similar to those of Schoen and Yau [33] in their study of higher-dimensional manifolds of positive scalar curvature. We also mention that, although topological censorship holds in arbitrary dimension, the techniques used in [12] to study the topology of black holes in $3+1$ dimensions via topological censorship do not extend directly to higher dimensions (see [13] for a further discussion of this point).

In section 3 we obtain a higher-dimensional analogue of the entropy bound (1). Given the role that scalar curvature plays in Hawking's argument, and in the higher-dimensional arguments of section 2, one is led to consider the so-called $\sigma$-constant (or Yamabe invariant) $[2,31]$ as the relevant topological invariant in higher dimensions. With regard to this choice we were also influenced by suggestive comments of Gibbons [16]. The $\sigma$-constant is a fundamental topological invariant of smooth compact manifolds, which we now briefly describe (see [2] for further details).

Let $\Sigma^{n-1}, n \geqslant 3$, be a smooth compact (without boundary) $(n-1)$-dimensional manifold. If $g$ is a Riemannian metric on $\Sigma^{n-1}$, let $[g]$ denote the conformal class of $g$. The Yamabe constant with respect to $[g]$, which we denote by $\lambda[g]$, is the number

$$
\lambda[g]=\inf _{\tilde{g} \in[g]} \frac{\int_{\Sigma} S_{\tilde{g}} \mathrm{~d} \mu_{\tilde{g}}}{\left(\int_{\Sigma} \mathrm{d} \mu_{\tilde{g}}\right)^{(n-3) /(n-1)}},
$$

where $S_{\tilde{g}}$ and $\mathrm{d} \mu_{\tilde{g}}$ are, respectively, the scalar curvature and volume measure of $\Sigma^{n-1}$ in the metric $\tilde{g}$. The expression involving integrals is just the volume-normalized total scalar curvature of $(\Sigma, \tilde{g})$. The solution to the famous Yamabe problem, due to Yamabe, Trudinger, Aubin and Schoen, guarantees that the infimum in (2) is achieved by a metric of constant scalar curvature.

The $\sigma$-constant of $\Sigma$ is defined by taking the supremum of the Yamabe constants over all conformal classes,

$$
\sigma(\Sigma)=\sup _{[g]} \lambda[g]
$$

As observed by Aubin, the supremum is finite, and, in fact, bounded above in terms of the volume of the standard unit $(n-1)$-sphere $S^{n-1} \subset \mathbb{R}^{n}$. The $\sigma$-constant divides the family of compact manifolds into three classes according to: (1) $\sigma(\Sigma)>0,(2) \sigma(\Sigma)=0$ and (3) $\sigma(\Sigma)<0$. It follows from the solution of the Yamabe problem that $\sigma(\Sigma)>0$ if and only if $\Sigma$ admits a metric of positive scalar curvature.

In the case $\operatorname{dim} \Sigma=2$, the Gauss-Bonnet theorem implies $\sigma(\Sigma)=4 \pi \chi(\Sigma)=8 \pi(1-g)$. In a certain sense, then, one may view the $\sigma$-constant as a generalization of the Euler characteristic to higher dimensions. This has been especially emphasized in three dimensions by Anderson, who in [2] describes some fundamental connections between the $\sigma$-constant and Thurston's geometrization conjecture for 3-manifolds. In this context there are some well known results and conjectures concerning the sign of the $\sigma$-constant. As noted above, $\sigma(\Sigma) \leqslant 0$ if and only if $\Sigma$ does not carry a metric of positive scalar curvature; some large classes of examples for which this holds are discussed in section 2 . It is believed that all compact hyperbolic 3-manifolds (which, in fact, account for most compact 3-manifolds) have a strictly negative $\sigma$-constant, but this has yet to be proven. There has been progress in determining the sign of the $\sigma$-constant in four dimensions; see, for example, the paper of Lebrun [25], in which Seiberg-Witten theory is used to determine the sign of the $\sigma$-constant within the class of compact complex algebraic surfaces (of, hence, four real dimensions) in terms of the Kodaira dimension. Finally, we mention that the $\sigma$-constant has also arisen in the cosmological context (see, for example, [3,9]; related notions have appeared in some earlier papers, as well, cf $[15,28])$. 
Let $\Sigma^{n-1}$ be a smooth black hole boundary contained in a spacelike hypersurface $V^{n}$ in a spacetime $M^{n+1}$, as described in section 2, and suppose $\sigma(\Sigma) \leqslant 0$ (which, in higher dimensions, is roughly analogous to the assumption $g \geqslant 1$ in the $\operatorname{dim} \Sigma=2$ case). Then the results in section 3 imply, under appropriate circumstances (e.g. $T_{00} \geqslant 0$ and $\Lambda<0$ ), that the area of $\Sigma^{n-1}$ satisfies,

$$
\operatorname{vol}\left(\Sigma^{n-1}\right) \geqslant\left(\frac{|\sigma(\Sigma)|}{2|\Lambda|}\right)^{(n-1) / 2},
$$

in analogy with the entropy bound (1).

We proceed to a detailed presentation of our results. Some concluding remarks are made in section 4.

\section{Topological restrictions}

We now describe the basic setting for our results. Let $\tilde{V}^{n}$ be a smooth spacelike hypersurface in a spacetime $M^{n+1}$, and let $\Sigma^{n-1}$ be a smooth compact hypersurface in $\tilde{V}^{n}$. To simplify certain statements and arguments, we assume that $\Sigma^{n-1}$ is connected. However, all of the results presented here apply to each component of $\Sigma^{n-1}$, in cases where it is not connected. Physically, one is to think of $\Sigma^{n-1}$ as the intersection of $\tilde{V}^{n}$ with the black hole event horizon in a stationary or static black hole spacetime. Alternatively, for dynamic black holes, one may think of $\Sigma^{n-1}$ as an apparent horizon contained within the black hole region. In either case, we assume that $\Sigma^{n-1}$ is marginally outer trapped, i.e. that the null expansion $\theta$ along $\Sigma^{n-1}$, with respect to the outward null normals, vanishes. Implicit in this assumption is that $\Sigma^{n-1}$ separates $\tilde{V}^{n}$ into an 'inside' and an 'outside'. Let $V^{n}$ denote the outside of $\tilde{V}^{n}$ together with $\Sigma^{n-1}$; hence, $V^{n}$, in the induced metric, is a smooth Riemannian manifold-with-boundary, with compact boundary $\Sigma^{n-1}$. Although some of our results will hold in more generality, for simplicity we are going to restrict attention to the time-symmetric case. In particular, our results apply in a natural way to the hypersurfaces of orthogonality in static black hole spacetimes. Some comments concerning the non-time-symmetric case are presented in section 4.

Thus, we assume that $V^{n}$ is a hypersurface of time symmetry, i.e. is totally geodesic. Then, as is standard in this case, conditions concerning the null expansion scalar along a hypersurface $W^{n-1}$ in $V^{n}$ can be expressed in terms of the mean curvature of $W^{n-1}$ as a submanifold of $V^{n}$. In particular, the assumption that $\Sigma^{n-1}$ is marginally outer trapped, reduces to the assumption that $\Sigma^{n-1}$ is a minimal surface (i.e. has vanishing mean curvature, $H=0$ ) in $V^{n}$. Similarly, an outer trapped surface in $V^{n}$ is a compact hypersurface $W^{n-1}$ in $V^{n}$ homologous to $\Sigma^{n-1}$ which has negative mean curvature, $H<0$, with respect to its outward unit normal in $V^{n}$. (By 'outward normal', we mean the normal to $W^{n-1}$ which points out from the region bounded by $\Sigma^{n-1}$ and $W^{n-1}$. By our sign conventions, $H=\operatorname{div}_{W} N$, where $N$ is the outward-pointing unit normal to $W^{n-1}$ in $V^{n}$.)

We say that $V^{n}$ is regular if, in addition to $\Sigma^{n-1}$ being minimal, there are no outer trapped surfaces in the interior of $V^{n}$. The non-existence of outer trapped surfaces in the domain of outer communications of a black hole spacetime, which we have incorporated into our model, is a standard result of black hole theory as developed in [21]. The arguments involved in establishing this do not depend on the dimension in an essential way. In all of our results it is assumed that $V^{n}$ is regular (or else satisfies a slightly stronger condition which we describe later).

Since $V^{n}$ is totally geodesic, the scalar curvature $S$ of $V^{n}$ is geometrically constrained by the Gauss equations to satisfy

$$
S=2 R_{00}+R
$$


where $R_{00}$ is the Ricci curvature of spacetime in the direction orthogonal to $V^{n}$ and $R$ is the scalar curvature of spacetime. In this paper we are primarily concerned with physical theories on spacetime whose field equations include equations of the form

$$
R_{\mu \nu}-\frac{1}{2} R g_{\mu \nu}+\Lambda g_{\mu \nu}=T_{\mu \nu}
$$

where $T_{\mu \nu}$ includes various matter field terms, and $\Lambda$ is constant. This includes general relativity, of course, but also various string field theories at sufficiently low energies (cf [23,29] and references cited therein). In particular, our results apply to various black string and black $p$-brane solutions, as well as to conventional higher-dimensional black holes. Taking into account the field equations, $S$ is constrained to satisfy

$$
S=2 T_{00}+2 \Lambda \text {. }
$$

Our first result establishes restrictions on the topology of the horizon $\Sigma^{n-1}$ under the assumption that $S=2 T_{00}+2 \Lambda$ is non-negative. (We have in mind, in particular, the asymptotically flat case, in which $\Lambda=0$ and the energy condition, $T_{00} \geqslant 0$, holds.) Under this assumption it is shown that $\Sigma^{n-1}$ carries (generically) a metric of positive scalar curvature. One may then appeal to the vast literature of results establishing restrictions on the topology of compact manifolds that admit metrics of positive scalar curvature (cf [18] for an overview). Particularly strong results hold in the $4+1(\operatorname{dim} \Sigma=3)$ case. We will recall some of these results after the proof of the following theorem.

Theorem 1. Let $V^{n}, n \geqslant 3$, be a regular time-symmetric spacelike hypersurface, with compact boundary horizon $\Sigma^{n-1}$, in a spacetime $M^{n+1}$, as described above. Suppose the scalar curvature of $V^{n}$ satisfies, $S=2 T_{00}+2 \Lambda \geqslant 0$ along $\Sigma^{n-1}$. Then either

(a) $\Sigma^{n-1}$ admits a metric of positive scalar curvature, or

(b) $S=2 T_{00}+2 \Lambda \equiv 0$ on $\Sigma^{n-1}$, and $\Sigma^{n-1}$ is totally geodesic and Ricci flat.

In the $3+1(\operatorname{dim} \Sigma=2)$ case, the theorem implies, using Gauss-Bonnet, that $\Sigma^{2}$ is either a flat torus or a topological sphere, in agreement with standard results [19-21]. (We recall that the torus arises as a borderline case in Hawking's original arguments, as well. The torus case is not easily eliminated without further assumptions, cf the discussion in [11].)

Proof. The proof is similar in spirit to the proof of Hawking's black hole topology theorem in $3+1$ dimensions [19,21], and its extension to apparent horizons [20], attributed to Gibbons. However, these proofs use the Gauss-Bonnet theorem to control the total scalar curvature of the horizon. This does not work in higher dimensions. Instead, we use a variation of the approach taken by Schoen and Yau [33] in their study of manifolds of positive scalar curvature in higher dimensions.

Let $t \rightarrow \Sigma_{t}$ be a variation of $\Sigma_{0}=\Sigma$ in $V^{n}$, with variation vector field $X=\phi N$, where $N$ is the unit normal along $\Sigma$ pointing into $V^{n}$ and $\phi$ is a smooth positive function on $\Sigma$. For $t \geqslant 0$ sufficiently small, $\left\{\Sigma_{t}\right\}$ foliates a neighbourhood of $\Sigma$. Extend $N$ to be the unit normal field to these $\Sigma_{t}$. For each $t$, let $H=H_{t}$ be the mean curvature of $\Sigma_{t}$; by our conventions, $H=\operatorname{div}(N)$. Then by Raychaudhuri's equation for a (possibly) non-geodesic unit vector field, which is valid for Riemannian, as well as Lorentzian, manifolds of arbitrary dimension, we have

$$
N(H)=-\operatorname{Ric}(N, N)-|B|^{2}+\operatorname{div}\left(\nabla_{N} N\right),
$$

where $\nabla$ and Ric are, respectively, the Levi-Civita connection and Ricci tensor of $V^{n}$, and $B=B_{t}$ is the second fundamental form of $\Sigma_{t}$ : for $X, Y \in T_{p} \Sigma_{t}, B(X, Y)=\left\langle\nabla_{X} N, Y\right\rangle$. 
The Gauss equations may be used to obtain the following 'rearrangement' $[14,32,33]$ of the first two terms on the right-hand side of (8),

$$
\operatorname{Ric}(N, N)+|B|^{2}=-\frac{1}{2} \hat{S}+\frac{1}{2} S+\frac{1}{2}|B|^{2} \quad \text { along } \Sigma,
$$

where $\hat{S}$ is the scalar curvature of $\Sigma$ in the induced metric, and we have used the fact that $\Sigma$ is minimal $(H=0)$.

The divergence term in (8) may be written as

$$
\operatorname{div}\left(\nabla_{N} N\right)=\operatorname{div}_{\Sigma_{t}}\left(\nabla_{N} N\right)-\left|\nabla_{N} N\right|^{2} .
$$

A further computation shows

$$
\nabla_{N} N=-\frac{1}{\phi} \nabla \phi \quad \text { along } \Sigma,
$$

where $\nabla \phi=\operatorname{grad}_{\Sigma} \phi$. Substitution of (11) into (10) gives

$$
\operatorname{div}\left(\nabla_{N} N\right)=-\frac{1}{\phi} \triangle \phi \quad \text { along } \Sigma,
$$

where $\Delta=\Delta_{\Sigma}$ is the Laplacian on $\Sigma$.

By substituting (9) and (12) into (8), and noting that along $\Sigma, \frac{\partial H}{\partial t}=X(H)=\phi N(H)$, we obtain,

$$
\begin{aligned}
\frac{\partial H}{\partial t} & =-\triangle \phi+\frac{1}{2}\left(\hat{S}-S-|B|^{2}\right) \phi \\
& =L(\phi) \quad \text { along } \Sigma,
\end{aligned}
$$

where $L=-\triangle+\frac{1}{2}\left(\hat{S}-S-|B|^{2}\right)$ is the so-called stability operator, rearranged according to (9).

Let $\lambda_{1}$ denote the first eigenvalue of $L$, and let $\phi$ be an associated eigenfunction,

$$
L(\phi)=\lambda_{1} \phi
$$

It is well known that for operators of the form of $L, \phi$ can be chosen to be strictly positive, $\phi>0$.

We observe that $\lambda_{1}$ cannot be negative; otherwise (13) and (14) would imply that $\frac{\partial H}{\partial t}<0$ along $\Sigma$. Since $H=0$ along $\Sigma$, this would mean that for $t$ sufficiently small, $\Sigma_{t}$ would be outer trapped, contrary to our assumptions.

Hence, $\lambda_{1} \geqslant 0$. This implies [10] that $\Sigma$ is a stable minimal hypersurface, by which we mean that $A^{\prime \prime}(0) \geqslant 0$ with respect to every variation $t \rightarrow \Sigma_{t}$ of $\Sigma_{0}=\Sigma$, where $A(t)=$ the area of $\Sigma_{t}$. The conclusion of theorem 1 then follows from arguments in [33]. Here we give a more direct argument, which is also relevant to the non-time-symmetric case. Let $g$ denote the induced metric on $\Sigma$, and consider the conformally related metric $\tilde{g}=\phi^{2 /(n-2)} g$. The scalar curvature $\tilde{S}$ of $\Sigma$ in the metric $\tilde{g}$ is given by

$$
\begin{aligned}
\tilde{S} & =\phi^{-n /(n-2)}\left(-2 \Delta \phi+\hat{S} \phi+\frac{n-1}{n-2} \frac{|\nabla \phi|^{2}}{\phi}\right) \\
& =\phi^{-2 /(n-2)}\left(2 \lambda_{1}+S+|B|^{2}+\frac{n-1}{n-2} \frac{|\nabla \phi|^{2}}{\phi^{2}}\right)
\end{aligned}
$$

where, for the second equation, we have used (14).

Equation (15) and the assumption $S \geqslant 0$ imply that $\tilde{S} \geqslant 0$. If $\tilde{S}>0$ at some point, then by well known results of Kazdan and Warner [24], one can conformally change $\tilde{g}$ (and, hence 
$g$ ) to a metric of strictly positive scalar curvature. Case (a) of theorem 1 then holds. If $\tilde{S}$ vanishes identically, then, by equation (15), $\lambda_{1}=0, S \equiv 0$ along $\Sigma, B=0$, and $\phi$ is constant. Equation (14) then implies that $\hat{S} \equiv 0$. By a result of Bourguinon (see [24]), it follows that $\Sigma$ carries a metric of positive scalar curvature, unless it is Ricci flat. Theorem 1 now follows.

Remark. As shown in the proof, the absence of outer trapped surfaces in $V$ implies that $\Sigma$ is stable. We make use of this observation, which is valid in the time-symmetric case only, in section 3 . In section 4 we consider the extension of theorem 1 to the non-time-symmetric case.

Theorem 1 asserts that, apart from exceptional cases, $\Sigma^{n-1}$ carries a metric of positive scalar curvature. (In fact, later we will show, under slightly stronger conditions, that case (b) cannot occur.) Although the scalar curvature is a rather weak geometric invariant, there are many known topological obstructions to the existence of metrics of positive scalar curvature. In the celebrated paper of Lichnerowicz [26], spinorial methods and the Atiyah-Singer index theorem were used to prove that compact $4 k$-dimensional spin manifolds of positive scalar curvature have vanishing $\hat{A}$-genus. This was followed later by work of Hitchin [22], who also used the spinorial method to obtain further vanishing theorems. However, these results left open the question as to whether, for example, the $k$-torus admits a metric of positive scalar curvature. Then in [32], Schoen and Yau proved that the fundamental group of a compact orientable 3manifold that admits a metric of positive scalar curvature cannot contain a subgroup isomorphic to a non-trivial surface group. This implies, in particular, that the 3-torus does not admit a metric of positive scalar curvature. In [33], Schoen and Yau generalized their techniques to higher dimensions, thereby establishing the existence of a large class of compact manifolds, including tori, of dimension up to seven, that do not admit metrics of positive scalar curvature. These results were put in a somewhat broader context, in the case of spin manifolds, by Gromov and Lawson [17,18] via the development of the notion of enlargability. We mention just two results obtained in [18]. It is proved there, for example, that any compact manifold (of arbitrary dimension) that admits a metric of positive scalar curvature, cannot carry a metric of non-positive sectional curvature $K \leqslant 0$. This result rules out many obvious topologies, such as products of tori and higher-genus surfaces, etc.

Now restrict attention to the case $\operatorname{dim} \Sigma=3$, and assume, by passing to a double cover if necessary, that $\Sigma$ is orientable. According to the prime decomposition theorem of Milnor, any such manifold $\Sigma$ can be expressed as a finite connected sum of three types of manifolds: (a) manifolds covered by homotopy 3-spheres (or 3-spheres, if the Poincaré conjecture holds); (b) manifolds diffeomorphic to $S^{2} \times S^{1}$; and (c) $K(\pi, 1)$ manifolds. Recall that a $K(\pi, 1)$ manifold is a manifold whose universal cover is contractible (such as the torus). In [18] it is shown that if $\Sigma$ admits a metric of positive scalar curvature then it cannot have any $K(\pi, 1)$ in its prime decomposition.

We are led to the following corollary.

Corollary 2. Let $V^{4}$ be a regular time-symmetric spacelike hypersurface, with compact boundary horizon $\Sigma^{3}$, in a spacetime $M^{4+1}$. Suppose the scalar curvature of $V^{4}$ satisfies, $S=2 T_{00}+2 \Lambda \geqslant 0$ along $\Sigma^{3}$. Then, unless it is flat, $\Sigma^{3}$ (or a double cover, if $\Sigma^{3}$ is nonorientable) is diffeomorphic to a finite connected sum of homotopy 3-spheres, perhaps with identifications, and $k \geqslant 0$ copies of $S^{2} \times S^{1}$.

Thus, the basic black hole topologies for the $(4+1)$-dimensional black hole spacetimes modelled in corollary 2 are $S^{3}$ (perhaps with identifications) and $S^{2} \times S^{1}$. If $\Sigma$ is flat then it must be a 3-torus, or be covered by a 3-torus. The corollary follows immediately from theorem 1 , 
the result of Gromov and Lawson described above, and the fact that in three dimensions, Ricci flat implies flat.

As mentioned earlier, case (b) of theorem 1 can be eliminated under slightly stronger conditions. Let $V^{n}$ be an orientable time-symmetric spacelike hypersurface with compact minimal $(H=0)$ boundary horizon $\Sigma^{n-1}$. A marginally outer trapped surface in $V^{n}$ is a compact minimal hypersurface in $V^{n}$ homologous to $\Sigma^{n-1}$. We say that $V^{n}$ is strongly regular provided: (1) there are no marginally outer trapped surfaces in the interior of $V^{n}$ and (2) there exists a compact hypersurface $\Sigma_{0}^{n-1}$ in $V^{n}$ homologous to $\Sigma^{n-1}$ which is mean convex, i.e. which satisfies $H>0$ with respect to the outward normal. Condition (2) is a mild asymptotic condition generally satisfied by asymptotically flat and asymptotically locally anti-de Sitter spacetimes. The non-existence of marginally outer trapped surfaces in the domain of outer communications is a standard result of black hole theory as developed in [21].

Theorem 3. Let $V^{n}, 3 \leqslant n \leqslant 7$, be a strongly regular time-symmetric spacelike hypersurface, with compact boundary horizon $\Sigma^{n-1}$, in a spacetime $M^{n+1}$, as described above. Suppose the scalar curvature of $V^{n}$ satisfies, $S=2 T_{00}+2 \Lambda \geqslant 0$. Then $\Sigma^{n-1}$ admits a metric of positive scalar curvature.

Proof. Let $V_{0}$ be the region in $V$ bounded by $\Sigma$ and $\Sigma_{0}$. $V_{0}$ is a compact Riemannian manifold-with-boundary, with (weakly) mean convex boundary $\partial V_{0}=\Sigma \cup \Sigma_{0}$. $\Sigma$ determines a non-trivial homology class in $H_{n-1}\left(V_{0}, \mathbb{Z}\right)$. We claim that $\Sigma$ is of least area in its homology class. To see this, we minimize area in the homology class of $\Sigma$. By standard results in geometric measure theory, there exists a smooth minimizer contained in $V_{0} \backslash \Sigma_{0}$; for the regularity of the minimizer we are using the assumption $n \leqslant 7$. Let $\Sigma_{1}$ denote a component of this minimizer; $\Sigma_{1}$ is necessarily minimal, i.e. has mean curvature $H=0$. To avoid having a marginally outer trapped surface contained in the interior of $V, \Sigma_{1}$ must meet $\Sigma$. However, then, since $\Sigma$ is also minimal, it follows from the maximum principle that $\Sigma_{1}=\Sigma$, which implies our claim.

In particular, $\Sigma$ is locally of least area, i.e. the area of $\Sigma$ is less than or equal to the area of any nearby surface isotopic to it. If the scalar curvature $S$ of $V$ were strictly positive, then arguments in [33] would imply that $\Sigma$, being locally of least area, would carry a metric of positive scalar curvature. If, as is our situation, the scalar curvature $S$ is merely assumed to be non-negative, it is shown in [6] (which generalizes to higher dimension results in [7]) that $\Sigma$ can fail to carry a metric of positive scalar curvature only under special circumstances: $\Sigma$ must be totally geodesic, and a neighbourhood $U$ of $\Sigma$ must be isometric to $[0, \epsilon) \times \Sigma$. Thus, to avoid the occurrence of marginally outer trapped surfaces in the interior of $V$, we conclude that $\Sigma$ carries a metric of positive scalar curvature.

Remark. The dimension restriction $n \leqslant 7$ is most likely an artefact of the proof. It only arises in showing that $\Sigma$ is locally of least area; if this is known to be the case, the dimension restriction is unnecessary. It is possible that the absence of outer trapped and marginally outer trapped surfaces alone is sufficient to imply that $\Sigma$ is locally of least area, but this does not follow immediately.

In the $4+1(\operatorname{dim} \Sigma=3)$ case, we obtain the following corollary to theorem 3 , similar to corollary 2.

Corollary 4. Let $V^{4}$ be a strongly regular time-symmetric spacelike hypersurface, with compact boundary horizon $\Sigma^{3}$, in a spacetime $M^{4+1}$. Suppose the scalar curvature of $V^{4}$ satisfies, $S=2 T_{00}+2 \Lambda \geqslant 0$. Then, $\Sigma^{3}$ (or a double cover, if $\Sigma^{3}$ is non-orientable) is 
diffeomorphic to a finite connected sum of homotopy 3-spheres, perhaps with identifications, and $k \geqslant 0$ copies of $S^{2} \times S^{1}$.

\section{Lower area bounds and the $\sigma$-constant}

With the setting as in the previous section, we obtain lower bounds for the area of the horizon $\Sigma^{n-1}$ in terms of its $\sigma$-constant, which is assumed to be non-positive, $\sigma(\Sigma) \leqslant 0$, under the assumption that $S=2 T_{00}+2 \Lambda$ satisfies $S \geqslant-\kappa$, where $\kappa$ is a positive constant. We have in mind, in particular, the asymptotically locally anti-de Sitter case, in which $\Lambda<0$ and the energy condition $T_{00} \geqslant 0$ holds.

Theorem 5. Let $V^{n}, n \geqslant 4$, be a regular time-symmetric spacelike hypersurface, with compact boundary horizon $\Sigma^{n-1}$, in a spacetime $M^{n+1}$, as in theorem 1 , such that $\sigma(\Sigma) \leqslant 0$. Suppose that the scalar curvature of $V^{n}$ satisfies, $S=2 T_{00}+2 \Lambda \geqslant-\kappa$, where $\kappa$ is a positive constant. Then the area of $\Sigma^{n-1}, \operatorname{vol}\left(\Sigma^{n-1}\right)$, satisfies,

$$
\operatorname{vol}\left(\Sigma^{n-1}\right) \geqslant\left(\frac{|\lambda[g]|}{\kappa}\right)^{(n-1) / 2} \geqslant\left(\frac{|\sigma(\Sigma)|}{\kappa}\right)^{(n-1) / 2},
$$

where $g$ is the induced metric on $\Sigma^{n-1}$.

In particular, if $T_{00} \geqslant 0$ and $\Lambda<0$, the area of $\Sigma^{n-1}$ satisfies the inequality (4).

Proof. By the remark following the proof of theorem $1, \Sigma$ is a stable minimal hypersurface, i.e. $A^{\prime \prime}(0) \geqslant 0$ with respect to every variation $t \rightarrow \Sigma_{t}$ of $\Sigma_{0}=\Sigma$, where $A(t)=$ the area of $\Sigma_{t}$. We recall the formula for the second variation of area with respect to variations $t \rightarrow \Sigma_{t}$ having variation vector field $X=\phi N$, where $N$ is the unit normal to $\Sigma$ pointing into $V$ and $\phi \in C^{\infty}(\Sigma)$

$$
A^{\prime \prime}(0)=\int_{\Sigma}\left(|\nabla \phi|^{2}-\left(\operatorname{Ric}(N, N)+|B|^{2}\right) \phi^{2}\right) \mathrm{d} \mu .
$$

Theorem 5 is now a consequence of the following purely Riemannian result, which may be of some independent interest.

Theorem 6. Let $V^{n}, n \geqslant 4$, be a Riemannian manifold with scalar curvature $S$ satisfying, $S \geqslant-\kappa$, where $\kappa$ is a positive constant. Let $\Sigma^{n-1}$ be a two-sided compact stable minimal hypersurface in $V^{n}$ with $\sigma$-constant $\sigma(\Sigma) \leqslant 0$. Then the area of $\Sigma^{n-1}$, $\operatorname{vol}\left(\Sigma^{n-1}\right)$, satisfies the inequalities in (16).

Proof. The second inequality in (16) follows immediately from the definition of the $\sigma$-constant. To prove the first inequality we use the following reformulation of the Yamabe constant [4]:

$$
\lambda[g]=\inf _{\phi \in C^{\infty}(\Sigma), \phi>0} \frac{\int_{\Sigma}\left([4(n-2) /(n-3)]|\nabla \phi|^{2}+\hat{S} \phi^{2}\right) \mathrm{d} \mu}{\left(\int_{\Sigma} \phi^{2(n-1) /(n-3)} \mathrm{d} \mu\right)^{(n-3) /(n-1)}} .
$$

In view of equations (9) and (17) the stability of $\Sigma^{n-1}$ implies that the following inequality holds for all $\phi \in C^{\infty}(\Sigma)$ (we may assume $\phi>0$ ):

$$
\int_{\Sigma}\left(|\nabla \phi|^{2}+\frac{1}{2}\left(\hat{S}-S-|B|^{2}\right) \phi^{2}\right) \mathrm{d} \mu \geqslant 0
$$


and hence,

$$
\int_{\Sigma}\left(2|\nabla \phi|^{2}+\hat{S} \phi^{2}\right) \mathrm{d} \mu \geqslant \int_{\Sigma} S \phi^{2} \mathrm{~d} \mu .
$$

Then, noting that $2<\frac{4(n-2)}{n-3}$, we obtain,

$$
\begin{aligned}
\int_{\Sigma}\left(\frac{4(n-2)}{n-3}|\nabla \phi|^{2}+\hat{S} \phi^{2}\right) \mathrm{d} \mu & \geqslant \int_{\Sigma} S \phi^{2} \mathrm{~d} \mu \\
& \geqslant-\kappa \int_{\Sigma} \phi^{2} \mathrm{~d} \mu .
\end{aligned}
$$

By Hölder's inequality,

$$
\int_{\Sigma} \phi^{2} \mathrm{~d} \mu \leqslant\left(\int_{\Sigma} \phi^{2(n-1) /(n-3)} \mathrm{d} \mu\right)^{(n-3) /(n-1)}\left(\int_{\Sigma} 1 \mathrm{~d} \mu\right)^{2 /(n-1)},
$$

which, when combined with (21) gives

$$
\frac{\int_{\Sigma}\left([4(n-2) /(n-3)]|\nabla \phi|^{2}+\hat{S} \phi^{2}\right) \mathrm{d} \mu}{\left(\int_{\Sigma} \phi^{2(n-1) /(n-3)} \mathrm{d} \mu\right)^{(n-3) /(n-1)}} \geqslant-\kappa(\operatorname{vol}(\Sigma))^{2 /(n-1)} .
$$

Making use of this inequality in (18) yields,

$$
\lambda[g] \geqslant-\kappa(\operatorname{vol}(\Sigma))^{2 /(n-1)},
$$

from which the first inequality in theorem 6 follows.

Theorem 6 is a higher-dimensional analogue of theorem 3 in [30], as well as the result of Gibbons discussed in the introduction (in which the stability of the horizon was assumed).

\section{Final remarks}

Consider the setting of theorem 1, in the general, non-time-symmetric case. Let $t \rightarrow \Sigma_{t}$ be the variation of $\Sigma$ in the proof of the theorem. Let $U$ be the timelike future-pointing unit normal along $V$. Then $K=U+N$ is a null vector field along $V$ such that $\theta=\theta_{t}=\operatorname{div}_{\Sigma_{t}} K$ is the null expansion scalar along $\Sigma_{t}$. By a similar computation to that given in the proof of theorem 1 , one obtains the following generalization of equation (13):

$$
\begin{aligned}
\frac{\partial \theta}{\partial t}=-\Delta \phi+ & \frac{1}{2}\left(\hat{S}-2 T_{a b} U^{a} K^{b}-2 \Lambda-|\Theta|^{2}\right) \phi+\phi \operatorname{div}_{\Sigma}\left(\nabla_{N} U\right)^{P} \\
& +\phi\left|\nabla_{N} N\right|^{2}-\phi\left|\nabla_{N} N+\left(\nabla_{N} U\right)^{P}\right|^{2},
\end{aligned}
$$

along $\Sigma$, where we have used the assumption that $\Sigma$ is marginally outer trapped, $\theta=0$ along $\Sigma$. Here, $\Theta$ is the null second fundamental form of $\Sigma$ with respect to $K$, and $X^{P}$ denotes projection of the vector $X$ onto $\Sigma$. In the $(3+1)$-dimensional case, Hawking's arguments can cope with the 'bad terms' in the above, e.g. the divergence term gets integrated away; our method in higher dimensions cannot deal so easily with these terms. However, if the term $\left(\nabla_{N} U\right)^{P}$ vanishes along $\Sigma$, equation (24) reduces to a form that permits the arguments in the proof of theorem 1 to go through. Thus, whenever this term vanishes, e.g. if the second fundamental form of $V$ vanishes along $\Sigma$, or, more generally, if $\nabla_{N} U \propto N$ along $\Sigma$, then theorem 1 generalizes to the non-time-symmetric case.

Finally, we note that the arguments presented in this paper, as in the case of the standard $3+1$ results, as well, require the black hole boundary $\Sigma^{n-1}$ to be sufficiently smooth, $C^{2}$, say. Horizons, however, need not have this degree of regularity, in general. For issues regarding the regularity of horizons, see, for example, [8], and references cited therein. 


\section{Acknowledgments}

We wish to thank Bill Minicozzi for some helpful comments. GJG was supported in part by NSF grant no DMS-9803566.

\section{References}

[1] Aharony O, Gubser S, Maldacena J, Ooguri $\mathrm{H}$ and $\mathrm{Oz}$ Y 2000 Large $N$ field theories, string theory and gravity Phys. Rep. 323184

[2] Anderson M 1997 Scalar curvature and geometrization conjectures for 3-manifolds Comparison Geometry (1993 MSRI Workshop) (MSRI Publications vol 30) (Cambridge: Cambridge University Press) pp 49-82

[3] Anderson M 2000 On long-time evolution in general relativity and geometrization of 3-manifolds Preprint gr-qc/0006042

[4] Besse A L 1987 Einstein Manifolds (Berlin: Springer)

[5] Brill D R, Louko J and Peldán P 1997 Thermodynamics of (3 + 1)-dimensional black holes with toroidal or higher genus black horizons Phys. Rev. D 56 3600-10

[6] Cai M 2001 Volume minimizing hypersurfaces in manifolds of nonnegative scalar curvature Minimal Surfaces, Geometric Analysis and Symplectic Geometry (Advanced Studies in Pure Mathematics) ed K Fukaya, S Nishikawa and J Spruck (Tokyo: The Mathematical Society of Japan) to appear

[7] Cai M and Galloway G J 2000 Rigidity of area minimzing tori in 3-manifolds of nonnegative scalar curvature Commun. Anal. Geom. 8 565-73

[8] Chruściel P, Delay E, Galloway G J and Howard R 2001 Regularity of horizons and the area theorem Ann. H Poincaré 2 109-78

[9] Fischer A E and Moncrief V 1999 The Einstein flow, the $\sigma$-constant and the geometrization of 3-manifolds Class. Quantum Grav. 16 L79-87

[10] Fischer-Colbrie D and Schoen R 1980 The structure of complete stable minimal surfaces in 3-manifolds of nonnegative scalar curvature Commun. Pure Appl. Math. 33 199-211

[11] Galloway G J 1994 Least area tori, black holes and topological censorship Contemporary Mathematics vol 170 ed J K Beem and K Duggal pp 113-23

[12] Galloway G, Schleich K, Witt D and Woolgar E 1999 Topolgical censorship and higher genus black holes Phys. Rev. D 60104039

[13] Galloway G, Schleich K, Witt D and Woolgar E 2001 The AdS/CFT correspondence conjecture and topological censorship Phys. Lett. B $\mathbf{5 0 5} 255-62$

[14] Gibbons G W 1972 The time symmetric initial value problem for black holes Commun. Math. Phys. 27 87-102

[15] Gibbons G W 1987 The entropy and stability of the universe Nucl. Phys. B 292 784-92

[16] Gibbons G W 1999 Some comments on gravitational entropy and the inverse mean curvature flow Class. Quantum Grav. 16 1677-87

[17] Gromov M and Lawson B 1980 Spin and scalar curvature in the presence of the fundamental group Ann. Math. $111209-30$

[18] Gromov M and Lawson B 1983 Positive scalar curvature and the Dirac operator on complete Riemannian manifolds Publ. Math. IHES 58 83-196

[19] Hawking S W 1972 Black holes in general relativity Commun. Math. Phys. 25 152-66

[20] Hawking S W 1973 The event horizon Black Holes ed C DeWit and B DeWit (New York: Gordon and Breach)

[21] Hawking S W and Ellis G F R 1973 The Large Scale Structure of Space-Time (Cambridge: Cambridge University Press)

[22] Hitchin N 1974 Harmonic spinors Adv. Math. 14 1-55

[23] Horowitz G T 1992 The dark side of string theory: black holes and black strings Trieste Spring School on String Theory and Quantum Gravity Preprint hep-th/9210119

[24] Kazdan J and Warner F 1975 Prescribing curvatures Proc. Symp. Pure Math. 27 309-19

[25] LeBrun C 1999 Kodaira dimension and the Yamabe problem Commun. Anal. Geom. 7 133-56

[26] Lichnerowicz A 1963 Spineurs harmoniques C. R. Acad. Sci., Paris A-B 257 7-9

[27] Maldacena J 1998 The large $N$ limit of superconformal field theories and supergravity Adv. Theor. Math. Phys. 2 231-52

[28] Murchadha N Ó 1989 The Yamabe theorem and general relativity Proc. Centre Math. Anal. Austral. Nat. Univ. (Canberra) vol 19, pp 137-67

[29] Peet A 2000 TASI Lectures on Black Holes in String Theory TASI-99 Preprint hep-th/0008241

[30] Shen Y and Zhu S 1997 Rigidity of stable minimal hypersurfaces Math. Ann. 309 107-16 
[31] Schoen R 1987 Variational Theory for the Total Scalar Curvature Functional for Riemannian Metrics and Related Topics (Springer Lecture Notes in Mathematics vol 1365) (Berlin: Springer) pp 120-54

[32] Schoen R and Yau S T 1979 Existence of incompressible minimal surfaces and the topology of three dimensional manifolds of non-negative scalar curvature Ann. Math. 110 127-42

[33] Schoen R and Yau S T 1979 On the structure of manifolds with positive scalar curvature Manuscr. Math. 28 $159-83$

[34] Witten E 1998 Anti-de Sitter space and holography Adv. Theor. Math. Phys. 253

[35] Woolgar E 1999 Bounded area theorems for higher-genus black holes Class. Quantum Grav. 16 3005-12 